\title{
Especies útiles de la selva baja caducifolia en las dunas costeras del centro de Veracruz
}

\author{
Useful plants of tropical dry forest on the coastal dunes of \\ the center of Veracruz State
}

\author{
Patricia Moreno-Casasola1 y Krystina Paradowska1
}

\begin{abstract}
RESUMEN
La selva baja caducifolia es un ecosistema bajo fuerte presión por las actividades humanas. Se distribuye tanto sobre dunas costeras como tierra adentro. El trabajo tiene como objetivo identificar las especies de árboles y palmas nativos útiles que crecen en las selvas bajas caducifolias y acahuales sobre las dunas costeras. En el trabajo se aplicaron cuestionarios y se realizaron entrevistas entre pobladores de San Isidro y Colonia La Mancha, en la costa de Veracruz. Se preguntó acerca del uso de los árboles de la selva baja caducifolia. Entre las 55 especies registradas, las más mencionadas fueron Cedrela odorata, Diphysa robinioides, Enterolobium cyclocarpum, Bursera simaruba, Gliricidia sepium, Tabebuia rosea, Chrysobalanus icaco, Guazuma ulmifolia, Acacia cochliacantha, Brosimum alicastrum, Bumelia celastrina, Cocos nucifera, Maclura tinctoria, Piscidia piscipula, Spondias mombin y Ficus spp. Se registraron 18 usos, siendo los más frecuentes la construcción de casas (119 menciones), postes y cercas vivas (99), leña (72) y comestibles (48), aunque también hay muchos usos que son poco mencionados. Cuatro de los usos se refirieron a servicios ambientales que prestan las plantas en las dunas. La especie con mayor número de usos es Gliricidia sepium y Bursera simaruba, es la especie con más partes aprovechadas, seguida por Gliricidia sepium, Guazuma ulmifolia y Brosimum alicastrum. También se preguntó acerca de las partes del árbol más usadas, la frecuencia de uso y su venta y precio local. Con base en los resultados se propone un conjunto de especies para enriquecer los acahuales y pastizales de las dunas para proveer madera, forraje, etc., a los habitantes y para mantener la cubierta arbolada de las dunas.
\end{abstract}

PALABRAS CLAVE:

Acahuales, plantas útiles, reforestación, servicios ambientales, usos de la madera.

\begin{abstract}
The tropical dry forest is an ecosystem under strong human pressure. It is found both inland and on coastal dunes. The objective of this research is to identify native tree and palm species growing on dunes that are used by local communities. In this research we carried out surveys and interviews among residents of San Isidro and Colonia La Mancha, in the coast of Veracruz on the use of trees of the tropical dry forest on dunes. Among the 55 registered species, Cedrela odorata, Diphysa robinioides, Enterolobium cyclocarpum, Bursera simaruba, Gliricidia sepium, Tabebuia rosea, Chrysobalanus icaco, Guazuma ulmifolia, Acacia cochliacantha, Brosimum alicastrum, Bumelia celastrina, Cocos nucifera, Maclura tinctoria, Piscidia piscipula, Spondias mombin and Ficus spp. were mentioned by more people. Eighteen uses were recorded, being the most frequent construction of houses (119 mentions), posts for fences and live fences (99), firewood (72) and food (48). Four of the uses mentioned refer to environmental services provided by these plants in the dunes. The species with higher number of uses is Gliricidia sepium and Bursera simaruba is the species in which more parts are used, followed by Gliricidia sepium, Guazuma ulmifolia and Brosimum alicastrum. We also inquired about the parts of the tree being used, the frequency, their sale and local price. Based on the results we suggest a group of species that can be used to increase species tree richness in the secondary
\end{abstract}


growths and grassland on dunes, which will provide wood, forage, etc., to the local inhabitants and help maintain the tree cover of the dunes.

KEY WORDS:

Secondary growth, useful plants, reforestation, environmental services, enduses of wood.

\section{INTRODUCCIÓN}

En el mundo las plantas son utilizadas para fines muy diversos. Siempre ha habido un enorme interés por conocer los beneficios que se obtienen de éstas. Existen numerosos trabajos sobre la utilidad de las plantas herbáceas y leñosas, con una gran variedad de enfoques que se han traducido en el desarrollo de una vasta tradición etnobotánica. Destacan en México los trabajos de Caballero y Cortés (2001), Bye (1993), Casas et al. (1994) entre otros muchos quienes enfatizan el uso tradicional de las plantas por la población indígena del país. También existen trabajos que tratan sobre usos muy específicos como el de Avendaño y Acosta (2000) con especies arbóreas nativas del Estado de Veracruz utilizadas para cercas vivas, el trabajo con especies forestales de Pennington y Sarukhán (1998) y el trabajo de Niembro (1986) con árboles útiles.

Un tema actual de investigación es el conocimiento y uso que los habitantes locales hacen de los árboles en las zonas tropicales. Pinedo-Vásquez et al. (2005) encontraron que las comunidades ribereñas de la región de Loreto en Perú, han establecido reservas comunitarias y emplean $60 \%$ de las especies arbóreas muestreadas. En otra región totalmente distinta, en Morongo, Tanzania, se identificaron un total de 133 especies arborescentes en 31 familias, de las cuales $69 \%$ se consideraban útiles. Gustad et al. (2004) analizaron los productos no made- rables del bosque y su comercialización. Encontraron que eran especialmente relevantes en las épocas de secas y que muchos árboles tenían una variedad de usos. En la Reserva de la Biosfera Maya en Guatemala se identificaron más de 80 especies de árboles útiles (Mutchnick y Mccarthy, 1997). De las 459 especies leñosas muestreadas en las tierras bajas del Atlántico de Costa Rica, en comunidades de vegetación secundaria, $70 \%$ de ellas tenía por lo menos un uso (Chazdon y Coe, 1999). En Venezuela, Aranguren (2005) registró 287 plantas útiles (incluyendo herbáceas).

Hay una gran ausencia de trabajos sobre los usos de las plantas de las selvas bajas caducifolias en México y ninguno enfocado a estas comunidades sobre dunas costeras. En un bosque deciduo de Yucatán, Rico-Gray et al. (1991) reportaron 301 especies de árboles y arbustos en el bosque y en huertos familiares. Para la región del Valle de Tehuacán existen varios trabajos. Casas et al. (2001), con una flora de más de 2700 especies, registraron usos para 808 especies. En esta zona también se han investigado la utilización, manejo y domesticación de varias especies de cactáceas de estas selvas. Destacan los trabajos realizados para Opuntia y Stenocereus por Casas et al. (1999). También se analizaron los usos de 15 especies de arbustos/árboles del género Mimosa (Camargo-Ricalde y Dhillion, 2004).

En México, las selvas bajas caducifolias o bosques tropicales deciduos se distribuyen desde el nivel del mar hasta los $1700 \mathrm{msnm}$, formando parte de sistemas costeros y sierras interiores, tanto en la vertiente Pacífica como Atlántica. Sobre esta última existen únicamente tres grandes manchones, uno de los cuales corresponde al centro de Veracruz, en el área situada entre Nautla, 
Alvarado, Jalapa y Tierra Blanca (Rzedowski, 1979). Son ecosistemas que atraviesan una estación seca que puede durar de siete a ocho meses, lo cual provoca un déficit hídrico y de nutrimentos minerales disponibles para la vegetación, dando como resultado un ecosistema complejo y diverso (Bullock et al., 1995). La cobertura vegetal de la selva baja caducifolia ha disminuido considerablemente debido a la ganadería extensiva, la extracción de madera, la agricultura y los incendios forestales. La superficie deforestada estimada en 1992 para esta selva en México fue de 163,000 hectáreas (Rincón et al., 1999). Sin embargo, Masera et al. (1997) consideran que la deforestación de este tipo de vegetación es aún mayor, ya que alcanza tasas de más de 300 mil ha por año, debido a las actuales políticas de desarrollo rural y los problemas de tenencia de la tierra.

La cubierta de selva baja caducifolia del centro de Veracruz se extiende sobre los lomeríos de la sierra y también sobre las dunas costeras. En la sierra de Manuel Díaz, en el municipio de Actopan entre otros, la selva que se extiende sobre la sierra alcanza altitudes de 800 msnm, límite de su distribución debido al descenso de la temperatura mínima (Rzedowski, 1972) y una de las especies más llamativas es la pata de elefante (Beaucarnea inermes y $B$. recurvata), especies que desaparecen hacia las zonas bajas costeras. La flora y vegetación de estas selvas, específicamente en el municipio de Actopan, ha sido descrita por Acosta (1986), Castillo-Campos (2006), Castillo-Campos y Medina (2006) y Castillo-Campos y Travieso-Bello (2006). Los últimos tres trabajos se han enfocado más en la zona costera.

Los médanos o dunas son sistemas formados por montículos de arena, con condiciones ambientales estresantes para las plantas (Moreno-Casasola y Vázquez, 2006). Se localizan en todas las costas de México, pero las más grandes en altitud y extensión están en Veracruz, Tamaulipas y Oaxaca. Prestan importantes servicios ambientales como la protección de la costa durante eventos de huracanes, tormentas y marejadas, entre otros (Martínez et al., 2007). En las etapas avanzadas de estabilización están cubiertas por pastizales entremezclados con acahuales y manchones de selva baja caducifolia. Una considerable proporción de estos terrenos han sido repartidos como parte de la entrega de tierras a los ejidos durante la Reforma Agraria y hoy en día se usan principalmente para ganadería.

Hoy en día, en los ecosistemas costeros de Veracruz quedan pocos remanentes de estas selvas pues la mayor parte de las dunas ha sido transformada debido a la extracción de leña y el uso ganadero y están cubiertas por pastizales moteados de manchones de matorrales bajos y acahuales con elementos de selvas bajas. El único manchón conservado, de 0,96 ha de selva baja caducifolia sobre dunas costeras en el municipio de Actopan, se ubica en la reserva del Centro de Investigaciones Costeras La Mancha (CICOLMA) del Instituto de Ecología, A. C. En el resto de las dunas de la región centro de Veracruz, sólo quedan pequeños manchones de restos de selvas y acahuales.

El presente trabajo tiene importancia ya que analiza la flora útil de estos ecosistemas (con énfasis en los árboles y palmas) y en segundo lugar permite obtener una lista de especies para reforestar estos ambientes que satisfaga algunas demandas locales y, simultáneamente, mantenga la cubierta arbórea y con ello la estabilización de las dunas y la diversidad de flora y fauna local. 


\section{DESCRIPCIÓN DE LA ZONA DE ESTUDIO}

Se trabajó con los habitantes del Ejido de San Isidro (habitantes de la población que lleva el mismo nombre) y con los habitantes de la Colonia La Mancha, ubicada en el camino que lleva al Centro de Investigaciones Costeras La Mancha, en el municipio de Actopan en la zona central de la costa de Veracruz, al norte del Puerto de Veracruz. En la figura 1 se indica la ubicación de estas poblaciones y las dunas costeras de este municipio, las cuales abarcan una superficie de 1782 ha, con base en la categoría de dunas costeras que aparece en el Inventario Forestal Nacional Serie II (20002001). La fisiografía, la geomorfología y la vegetación han sido descritas por Geissert (2006), García Gil (2006) y Moreno-Casasola y Travieso-Bello (2006), respectivamente. El clima es cálido sub-húmedo, con un periodo lluvioso, de junio a septiembre (cae 78\% de la precipitación total anual, 1286,7 $\mathrm{mm})$, y otro seco, de octubre a mayo. La temperatura media anual varía de 21.1 a 27,3 ${ }^{\circ} \mathrm{C}$ (Travieso-Bello y Campos, 2006).

Los poblados de San Isidro y Colonia La Mancha están bordeados por grandes campos de dunas con sistemas móviles y estabilizados. En San Isidro viven 1316 personas y en la Colonia La Mancha 107 personas. Ruelas-Monjardín (2006) y Paradowska (2006) describen la situación socioeconómica de la zona costera de este municipio. Esta franja costera permaneció poco comunicada con el resto del estado hasta la década de los sesenta, cuando se construyó la carretera costera que parte de Cardel hacia Nautla. A partir de este momento se inició una mayor intervención del Estado en la infraestructura, culminando con la construcción de la central núcleoeléctrica Laguna Verde en el extremo norte de la zona, perteneciente ya al municipio de Alto Lucero. La ocupación principal de los habitantes es la ganadería, el cultivo de mango y papaya, la siembra de caña de azúcar en tierras de riego, los cultivos de autoconsumo en tierras de temporal (maíz, frijol, calabaza, chile), la pesca y la acuacultura. La prestación de servicios se manifiesta en pequeños comercios, restaurantes y transporte local. La migración a los Estados Unidos se torna, cada vez con mayor frecuencia, como una opción para incrementar el ingreso económico de la familia.

\section{OBJETIVOS}

El objetivo de este estudio fue identificar las especies útiles, específicamente arbóreas y palmas, tanto nativas como introducidas, que crecen silvestres formando parte tanto de las selvas bajas caducifolias como de los acahuales derivados de estas comunidades, cuando se modifica el uso del suelo sobre las dunas costeras. Se recabó información sobre cuáles son las especies útiles y el uso que se les da, cuáles son usadas con más frecuencia, cuáles son las partes de la planta más utilizadas y cómo es la comercialización de la madera en la zona. A largo plazo, la información permitirá conocer cuáles son las especies que la población local requiere así como el uso más frecuente y sentar las bases para la elaboración e instrumentación de programas de reforestación y enriquecimiento de acahuales, así como desarrollar plantaciones forestales multiespecíficas usando árboles nativos sobre dunas costeras. Esto permitirá satisfacer algunas demandas locales, así como mantener la cubierta arbórea y con ello, la estabilización de las dunas y la conservación de la diversidad local, garantizando el mantenimiento de los servicios ambientales que las dunas proporcionan. 


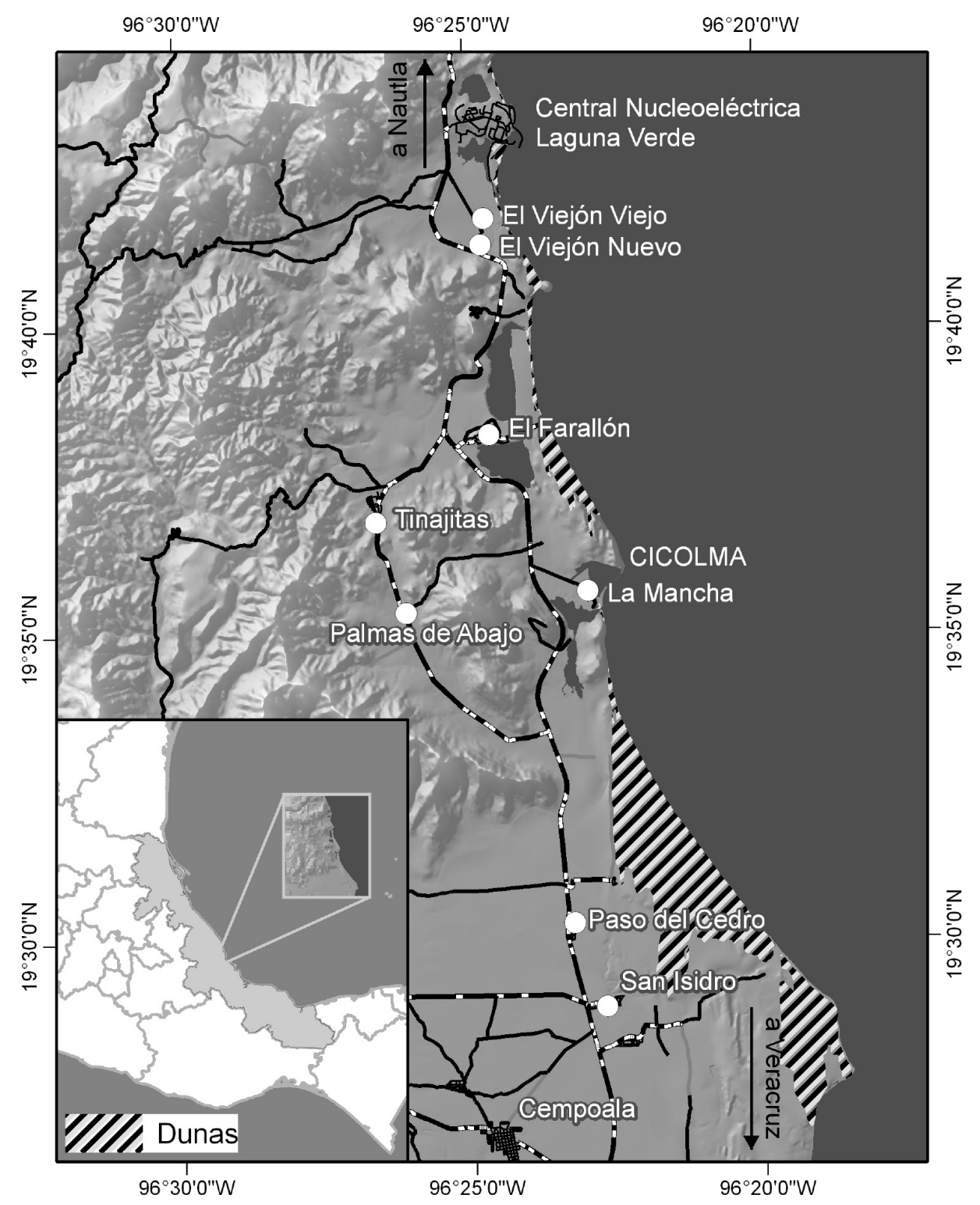

Figura 1. Mapa de la zona de trabajo donde se pueden ver las poblaciones, particularmente Colonia La Mancha y San Isidro, así como las dunas del Municipio de Actopan, Veracruz. 


\section{METODOLOGÍA}

La entrevista estandarizada abierta, constituida por un cuestionario de preguntas ordenadas en tres bloques temáticos, redactado y aplicado por igual a todos los entrevistados y de respuesta libre y abierta (Valles, 1997; Mejía y Sandoval, 2003), fue utilizada como base para la obtención de la información. El primer bloque fue dedicado a recabar datos de los entrevistados, el segundo a la percepción de la utilidad de los médanos, en el que también se pidió una lista de plantas útiles e importantes que se encuentran en este hábitat, y la tercera parte consistió en listados de 14 preguntas por cada especie de árbol o palma previamente mencionada como importante. Incluyó temas de nomenclatura local, las partes de las plantas usadas y su eventual comercialización y precio. El cuestionario utilizado se presenta en el anexo 1.

El cuestionario fue dirigido a los campesinos conocedores del tema de árboles y dunas y usuarios de estos ecosistemas, provenientes de ambas localidades. Para localizar a los informantes se emplearon redes personales y la técnica de "bola de nieve" (un entrevistado recomienda al siguiente y así sucesivamente) (Mejía y Sandoval, 2003). El cuestionario se aplicó a un total de 35 habitantes, 17 personas de Colonia La Mancha que representan $18,2 \%$ de las casas-habitación y 18 personas de San Isidro que representan $1,2 \%$. Los participantes fueron seleccionados debido a que poseen o trabajan en las parcelas ubicadas en los médanos de la zona de estudio. El menor porcentaje de personas con las que se trabajó en San Isidro se debe a que solamente unos cuantos habitantes tienen terrenos sobre las dunas, ya que la mayor parte son cañeros y poseen y trabajan tierras con otras características. La mayoría de los entrevistados fueron hombres (30 entre ambas localidades), ya que son quienes recorren habitualmente las parcelas ubicadas en el sitio de estudio y aprovechan la madera de distintos árboles como parte de sus labores productivas cotidianas. Son ejidatarios, pequeños propietarios y peones empleados en ranchos ganaderos, cultivos de caña de azúcar y milpas, por lo que tienen un contacto cotidiano con estos ecosistemas. Las cinco mujeres a las que se aplicó el cuestionario aportaron mayor información sobre los usos alimentarios y medicinales de las plantas. La edad de los entrevistados osciló entre 16 y 87 años (predominando las edades entre 36 y 40 y 56 a 65). Los datos recopilados se registraron en una hoja de cálculo para facilitar su análisis. El trabajo se acompañó de la observación directa así como de conversaciones y recorridos por las dunas junto con los informantes. Fue un recurso útil para la interpretación de los datos.

Para relacionar el nombre común con el nombre científico, se colectaron muestras de las plantas en el campo y se compararon con los ejemplares depositados en el herbario del Instituto de Ecología, A.C. (XAL) y con la bibliografía especializada (Pennington y Sarukhán, 1998; Castillo-Campos y Medina, 2002). Cuando había dudas se consultó con taxónomos expertos (G. CastilloCampos). Para enlistar los nombre científicos se utilizó el sistema de clasificación botánica de Cronquist (1988) y los autores de las especies se consultaron en el trabajo de Brummitt y Powell (1992).

\section{RESULTADOS}

Se mencionaron un total de 103 especies útiles creciendo en ambas localidades. De éstas, se eliminaron 15 que solamente eran cultivadas en huertos y no crecían sobre las dunas de manera silvestre, 14 que correspondían a hierbas o arbustos chicos, 13 que no se pudieron identificar y 
fueron mencionadas solo por una o dos personas y 6 que tenían dos o más nombres comunes resultando en información confusa. Por tanto, en el presente análisis se trabajó finalmente con una lista de 55 especies de árboles y arbustos creciendo sobre las dunas costeras de la región, que tienen algún uso o interés para los pobladores de la zona. Incluyen a tres especies de árboles introducidas (Cocos nucifera, Terminalia catappa y Casuarina equisetifolia). La lista de especies con su respectivo nombre científico, el nombre local, la familia, el número de veces que fueron mencionadas en los cuestionarios y los usos que se le dan, se muestra en el anexo 2. De las 55 especies registradas, únicamente 18 especies fueron mencionadas por más de cinco personas, 5 por cuatro personas, mientras que 17 especies fueron mencionadas por una sola persona (anexo 2). Las especies que fueron mencionadas con mayor frecuencia se consideran las de mayor importancia. Las especies que fueron más nombradas, en orden decreciente, son: Diphysa robinioides $(60$ menciones debido a los diferentes usos que se le dan), Gliricidia sepium (44), Cedrela odorata (39), Enterolobium cyclocarpum (34), Bursera simaruba (31), Tabebuia rosea (21), Guazuma ulmifolia (20), Brosimum alicastrum (18), Acacia cochliacantha (17), Maclura tinctoria (13), Casuarina equisetifolia (13), Spondias mombin (12), Chrysobalanus icaco (12), Karwinskia humboldtiana (10), Bumelia celastrina (10), Cordia dentata (9), Tabebuia chrysantha, Piscidia piscipula y Swietenia macrophylla (8 cada una), Acrocomia aculeata, Cocos nucifera, Lysiloma divaricata, Coccoloba barbadensis y Ficus spp. (6 cada uno).

Usos. Para cada especie se muestran los tipos o clases de usos que se les da a los árboles, palmas y los dos arbustos grandes registrados, y el número de especies que son utilizadas en cada tipo (anexo 2). Se detectaron 18 usos. Cuatro de estos usos realmente representan beneficios 0 servicios ambientales que los árboles prestan a la comunidad: funcionan como cortina de viento durante nortes, ayudan en la estabilización de arena evitando que ésta cubra cultivos y caseríos, brindan alimento y refugio para la fauna $y$ permiten el repoblamiento de árboles en la zona.

Los usos, bajo una concepción más utilitaria, reportados más frecuentemente son para construcción de casas (119 menciones), postes (71) y cercas vivas (37), leña (72), comestibles (48) y elaboración de muebles (36), aunque también hay muchos usos que son poco mencionados (para carbón, apicultura, ornamentales). Se encontró que el número de especies que se utilizan en cada práctica o tipo de uso es muy variable.

En el anexo 2 se muestra el número de especies que se utilizan en cada uno de los usos mencionados. Cada especie puede tener diversos usos, aunque la mayoría tiene menos de tres. Gliricida sepium se destaca por ser la especie con el mayor número de usos (10). Le siguen Guazuma ulmifolia (8), Enterolobium cyclocarpum (7), Brosimum alicastrum (6), Bursera simaruba (6), Maclura tinctoria (6), Cedrela odorata (5), Cordia dentata (5), Karwinskia humboldtiana (5), Spondias mombin (5), Tabebuia rosea (5) y Casuarina equisetifolia (5). Con solamente 4 usos se mencionaron Acacia cochliacantha, Diphysa robinioides, Coccoloba barbadensis y Swietenia macrophylla, mientras que con tres usos se mencionaron las palmas Acrocomia aculeata y Cocos nucifera y los árboles Tabebuia chrysantha, Piscidia piscipula, Lysiloma divaricata, Bumelia celastrina, Nectandra salicifolia, Thoinidium decandrum, Tecoma stans, Ficus spp. (no fue posible diferenciar a nivel de especie) y Zanthoxylum sp. 

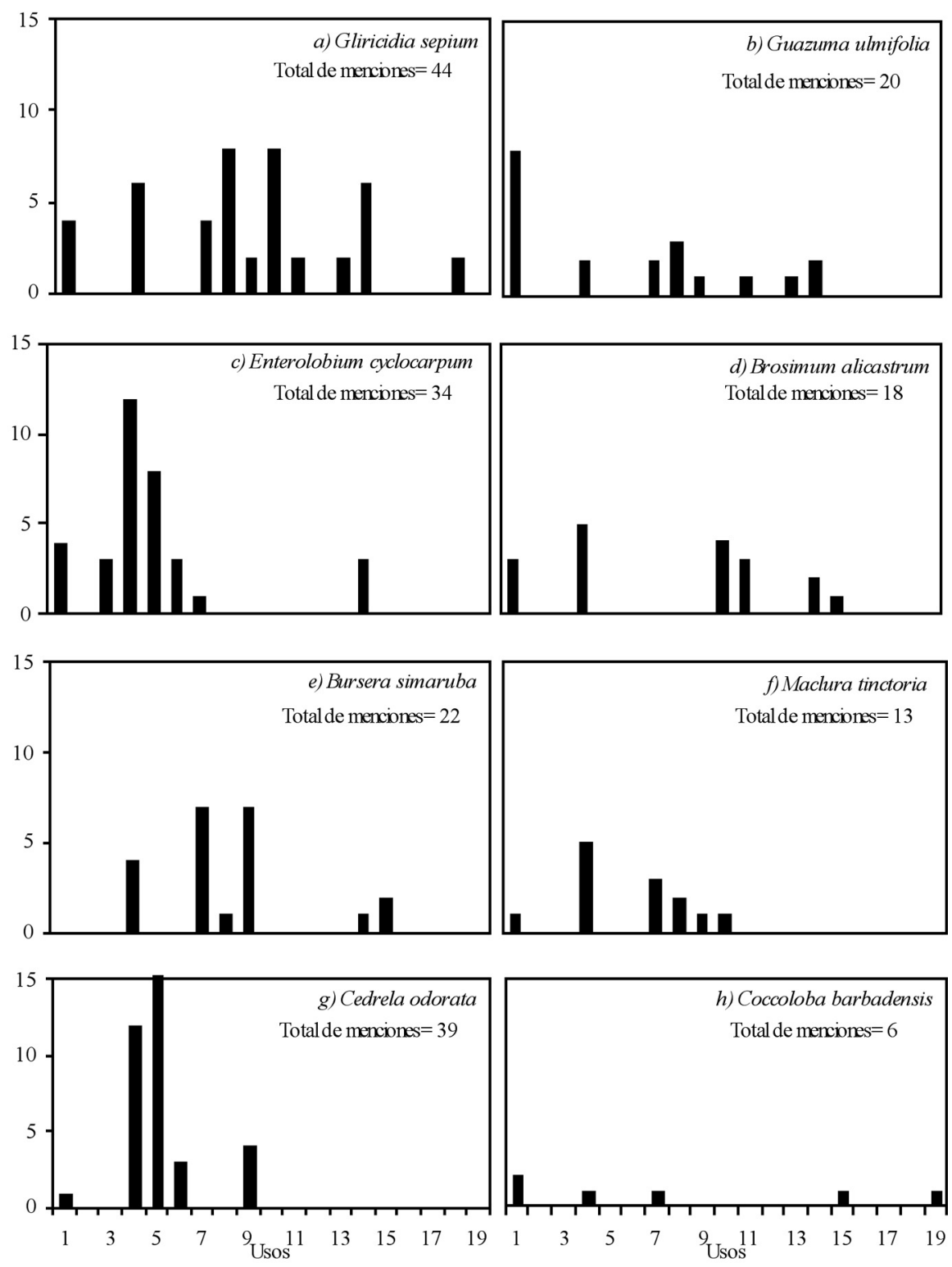

Figura 2. Usos de las especies nativas más utilizadas por los encuestados: leña=1, carbón $=2$, utensilios $=3$, construcción $=4$, muebles $=5$, botes y cayucos $=6$, postes para cerca $=7$, cercas vivas $=8$, medicinal $=9$, comestible $=10$, alimento ganado $=11$, ornamental $=12$, apicultura $=13$, sombra $=14$, alimento y refugio para fauna $=15$, cortina de viento $=16$, repoblamiento $=17$, estabilizar arena $=18$, no se usa $=19$. 
Para 12 especies se mencionaron dos usos y para 16 especies uno solo. Las especies reportadas con mayor número de usos se presentan en la figura 2 a-h.

Para la obtención de leña se emplean 29 especies, para construcción 25 , para postes en cercas 21 , como medicinales 16 , comestibles 15 , cercas vivas 12 , para sombra 10 , alimento y refugio de fauna 7, elaboración de utensilios 6 y muebles 4 (Anexo 2).

Partes utilizadas. Se registraron 380 menciones acerca de las partes de la planta que se usan. Las partes más utilizadas de los árboles fueron los troncos, las ramas y los frutos. $38 \%$ de las menciones fueron para el tronco (141 menciones) y ramas $77(20 \%)$. Hubo una sola mención para el uso de la raíz, de la savia y del cogollo (Figura 2a). Se utilizan los troncos de 33 especies, las ramas de 24 y los frutos de 18, las hojas y las semillas de 8. Aunque los pobladores indicaron que de 25 especies se usaban todas las partes, el término "todas" resultó algo ambiguo pues no siempre incluye todas las partes mencionadas en la figura 2 y 3 .

La tabla 1 muestra las especies de las cuales se usa un mayor número de partes y cuáles son. Bursera simaruba es

Tabla 1. Listado de las especies en las cuales hay un mayor número de estructuras o partes de la planta en uso. El valor indica el número de menciones al uso de esa parte de la planta por especie.

\begin{tabular}{|c|c|c|c|c|c|c|c|c|c|c|c|c|}
\hline Especie & 0 & 70 & $7 \mathrm{sin}$ & 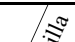 & ఫَ & \& & $\sqrt{4}$ & $\Delta$ & . & $T$ & ( & 0 \\
\hline Acacia cochliacantha & 5 & 1 & 2 & & & & & & & & & 2 \\
\hline Acroco mia aculeata & & 2 & & 2 & 2 & 2 & & & & & & 3 \\
\hline Attalea butyracea & & 1 & & 1 & 1 & & & & 1 & & & 3 \\
\hline Brosim um alicastrum & 1 & 5 & & 3 & 1 & 1 & & & & & & 5 \\
\hline Bumelia celastrina & & 2 & 1 & & & 4 & & & & & & 3 \\
\hline Bursera sima ruba & 2 & 4 & 1 & & & 1 & 1 & & 4 & 2 & & 7 \\
\hline Cas uarina equisetifolia & 7 & 3 & & & & & & & & & & 2 \\
\hline Cedrela odorata & 4 & 2 & 6 & & & & 4 & & & & & 4 \\
\hline Chryso balan us icaco & 1 & & 2 & 3 & 3 & 9 & & & & & & 4 \\
\hline Coccoloba barbadensis & 1 & 1 & & & & 1 & & & & & & 3 \\
\hline Cocos nucifera & & 2 & & 1 & 1 & 1 & & & 3 & & & 4 \\
\hline Cordia dentata & 1 & 4 & 3 & & & & & & & 1 & & 4 \\
\hline Diphysa robinioides & 1 & 25 & 12 & & & & & & & & & 3 \\
\hline Enterolobium cyclocarpum & 4 & 18 & 6 & & & & & & & & & 3 \\
\hline Ficus spp. & 3 & & 2 & & & & & & & & & 2 \\
\hline Gliricidia sepium & 7 & 7 & 8 & & 8 & & & & 4 & & & 5 \\
\hline Guazu ma ulm ifolia & 7 & 2 & & & 1 & 1 & & & 1 & & & 5 \\
\hline Kar win skia h umboldtiana & 1 & 3 & 2 & & & & & & & & & 3 \\
\hline Lysiloma divaricata & 1 & 1 & 1 & & & & & & & & & 3 \\
\hline Maclura tinctoria & 1 & 3 & 1 & & & 2 & & & & & & 4 \\
\hline Sabal mexicana & & 2 & & & & & & 1 & 2 & & & 3 \\
\hline Spondias mombin & & & 3 & & & 6 & & & & & 1 & 3 \\
\hline Swietenia ma cro phylla & & 4 & 1 & 1 & 1 & & & & & & & 3 \\
\hline Tab ebu ia crysan tha & & 4 & 1 & & & & & & & & & 2 \\
\hline Tabebuia rosea & & 13 & 7 & & & & 1 & & & & & 3 \\
\hline Tecoma stan $s$ & 1 & 2 & 2 & & & & & & & & & 3 \\
\hline Zan thoxylum sp. & 1 & 2 & 2 & & & & & & & & & 3 \\
\hline
\end{tabular}







la especie con más partes aprovechadas, seguida por Gliricidia sepium, Guazuma ulmifolia y Brosimum alicastrum. En la mayoría (12 especies) sólo se utiliza una parte, y en tres especies la utilización no involucra el uso directo de ninguna parte. Por ejemplo, Terminalia catappa, especie introducida, se usa como sombra.

Venta y precios. La mayoría de los pobladores (58\%) no comercializa con las plantas útiles y solamente $12 \%$ lo hace ocasionalmente. Pocas especies tienen valor de venta. A nivel de especies, $16 \%$ se comercializa, aunque sea ocasionalmente (Annona muricata, Byrsonima crassifolia, Cocos nucifera, Cordia sp., Gliricidia sepium, Karwinskia humboldtiana, Ocotea cernua, Sabal mexicana y Swietenia macrophylla). 4\% de las especies recibió respuestas mixtas de sí y no (Cedrela odorata y Diphysa robinioides), y $18 \%$ a veces (Acacia cochliacantha, Cordia dentata, Enterolobium cyclocarpum, Nectandra salicifolia, Piscidia piscipula, Spondias mombin, Tabebuia crysantha, Tabebuia rosea, Zanthoxylum kellermanii). $47 \%$ de las especies no tiene mercado o no se acostumbra su venta y $15 \%$ de las especies no tuvo respuesta. Trece de ellas se venden localmente entre los propios vecinos. Tres especies se venden con frecuencia tanto localmente como en las madererías (Swietenia macrophylla, Cedrela odorata, Enterolobium cyclocarpum). La tabla 2 indica algunas de las especies que se venden y el precio que tienen actualmente en la zona.

\section{DISCUSIÓN}

Las especies registradas e identificadas pertenecen a 30 familias. Las familias con mayor número de especies útiles son Mimosaceae (7), Moraceae (5), Arecaceae (4) y Bignoniaceae, Boraginaceae, Fabaceae y Rubiaceae con tres especies cada una. La mayoría de las especies mencionadas son silvestres, excepto Cocos nucifera, Terminalia catappa y Casuarina equisetifolia. Algunas de las especies silvestres que crecen en las dunas también se plantan en los huertos por ser comestibles, principalmente los zapotes (Manilkara zapota) y el jicaco (Chrysobalanus icaco).

La gran mayoría de las menciones se refieren a la utilización de los árboles o parte de los mismos. El principal aprove-

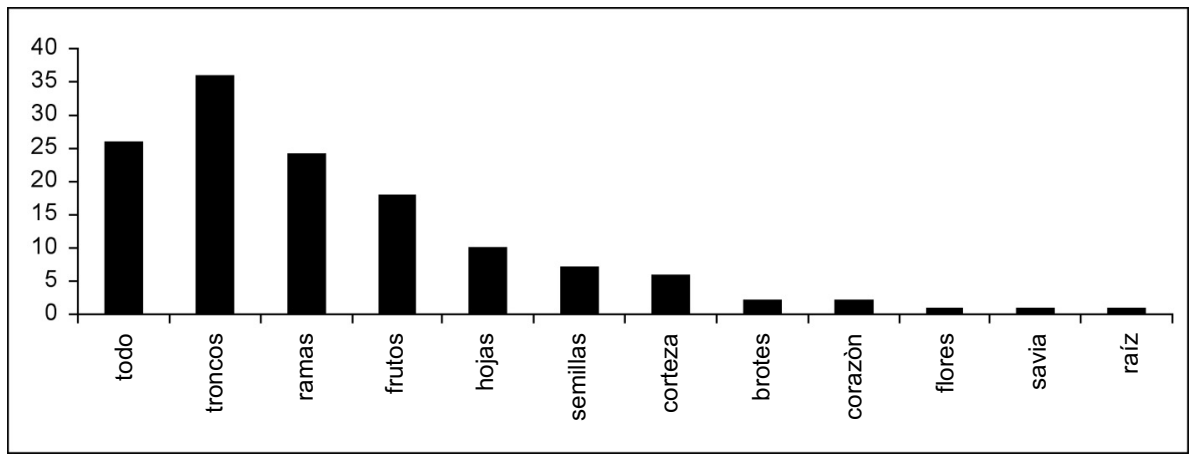

Figura 3. Número de menciones sobre las partes de la planta que son más usadas por los pobladores de la localidad. 
chamiento de los árboles y palmas distribuidos en la franja de médanos se realiza con el fin de obtener materiales para combustible de uso doméstico, construcción y postes para cercados. Significa que el uso de la madera sigue siendo fundamental para la vida diaria de estas comunidades, proporcionando techo, alimento y apoyando las actividades productivas. Ello explica porqué los troncos y ramas son las partes más usadas. Sin embargo, los comentarios de la gente durante las entrevistas revelaron una clara tendencia, al menos en el caso de las viviendas, hacia el desuso de madera de cedro y hoja de palmas para techar las casas, costumbre que ya casi no se observa en la zona. En el primer caso, los cedros que aún se encuentran están en terrenos privados, además de que se requieren permisos oficiales para su corte. También las maderas duras de palo de rabia (Karwinskia humboldtiana), moral (Maclura tinctoria), quebracha (Diphysa robinioides), aví (Piscidia piscipula) o flor de día (Tabebuia crysantha), tradicionalmente usadas para vigas y horcones, se emplean menos que antes.

En un territorio ganadero y agrícola como la zona de estudio (Travieso-Bello y Campos, 2006), el cercado de parcelas tiene una gran importancia, pues es necesario reemplazar algunas piezas podridas por nuevas, lo cual ocurre en promedio cada cinco años. Los entrevistados indicaron que los postes de maderas duras (Karwinskia humboldtiana, Gliricidia sepium, Diphysa robinioides, Piscidia piscipula, Tabebuia crysantha) aguantan hasta veinte o treinta años. El bajo costo de los postes para cercado con respecto a los de cemento hace que se sigan usando. También se usan los postes para crear cercas vivas aprovechando los árboles comunes de la zona cuyas ramas pegan fácilmente. Entre los más utilizados están Gliricidia sepium, Bursera simaruba, y en segundo lugar Spondias mombin y Cordia dentata (Anexo 2). Cabe decir que este tipo de aprovechamiento permite a los pobladores tener dos de las especies que más usos tienen y de las cuales usan más partes, directamente en sus zonas de trabajo.

El aprovechamiento para leña es otro de los usos más frecuentes. La lista de árboles usados es muy larga (Anexo 2, Figura 2), incluyendo árboles como los de madera muy dura, pero también cualquier árbol seco o bien ramas secas, y los que a decir de los pobladores "no sirven para otra cosa". El empleo actual de la leña se limita a la cocina. El uso de leña aumenta significativamente en toda esta región cañera durante la zafra, cuando grandes cantidades de comida se preparan para alimentar a los jornaleros. Los volúmenes de leña reportados por algunos entrevistados oscilan alrededor de una carga por cada una o dos semanas por hogar (Tabla 2), lo cual representa aproximadamente 40 y $50 \mathrm{~kg}$.

Como alimento se mencionaron 18 especies con frutos y 7 cuyas semillas tienen usos, aunque cada vez se emplean menos. Las frutas nativas como anona (Anonna muricata), pionche (Bumelia celastrina) y el jicaco (Chrysobalanus icaco), anteriormente consumidas frescas o preparadas en atole o en dulce ya casi no se consumen, al igual que el interior de la almendra de coyol (Attalea butyraceae) preparado como tortilla de coyol o en bocadillo dulce como cocada. La flor de cocuite (Gliricidia sepium) solía prepararse en tortitas con huevo o a la mexicana. Desapareció totalmente el consumo de las semillas de ojite (Brosimum alicastrum). Por temporadas, se consumen algunas plantas que crecen de manera silvestre en los médanos como los frutos silvestres de nanche (Byrsonima crassifolia), guayaba (Psidium guajava), ciruela (Spondias 
mombin) y jicaco (Chrysobalanus icaco), en ocasiones.

En la zona se siguen empleando las plantas como remedios medicinales caseros. El número de árboles usados es mucho más bajo que el reportado para otras zonas con mayor tradición indígena, tanto en México (Rico-Gray et al., 1991; Casas et al., 2001) como en otras partes de América (Chazdon y Coe, 1999; Aranguren, 2005). En los hogares aún se emplean remedios caseros para bajar la calentura, como es el caso de las hojas de palo mulato (Bursera simaruba). Esta fue la especie más mencionada por sus propiedades medicinales. Otro de los usos medicinales más arraigados es el emplear los frutos de Randia monantha para hacer un añejado de aguardiente como antídoto para el veneno de víboras, alacranes y capulinas, que se lleva cuando se va a trabajar en el campo. Se mencionaron las propiedades analgésicas de las cortezas de roble (Tabebuia rosea), cedro (Cedrela odorata), cucharo (Dendropanax arboreus) y cornezuelo (Acacia cornigera) que antes ayudaban a calmar el dolor de muelas. La corteza de la quina (Chinchona sp.) cayó en desuso por la erradicación de la malaria. La savia de la higuera negra (Ficus cotinifolia) se usa para falseaduras en animales domésticos.

La importancia vigente del uso de los árboles para leña y construcción ha sido descrito por otros muchos autores. Pinedo-Vásquez et al. (2005) trabajaron con comunidades ribereñas de Perú. Dividieron los usos registrados en seis categorías: alimento, construcción, utensilios, medicinales, comercio y la categoría de otros. También presentan y discuten las reglas que operan en la comunidad para el aprovechamiento de estos recursos. Luoga et al. (2000) en Tanzania, identificaron 51 especies útiles. Clasificaron los usos en 12 categorías y entre los princi- pales estaba el carbón de leña, la leña, los postes para construcción de casas y los usos medicinales y comestibles.

Otro de los usos más difundidos es el medicinal. Los números de especies reportados en la literatura generalmente rebasan los registrados en la zona. En bosques deciduos de Yucatán, Rico-Gray et al. (1991) reportaron que $73.7 \%$ de las 301 especies de árboles y arbustos en el bosque y en huertos familiares tenían al menos un uso. Las especies con más usos fueron: Bursera simaruba y Gliricidia sepium (10 usos), Cordia dodecandra (9), Plumeria rubra, Caesalpinia gaumeri, Vitex gaumeri, Enterolobium cyclocarpum y Piscidia piscipula (7). La mayoría $(69,7 \%)$ tenían uso medicinal, seguido por el uso apícola (39,9\%), alimenticio $(30,3 \%)$, como combustible $(17,4 \%)$, para construcción $(18,8 \%)$ y maderable $(11 \%)$. Gustad et al. (2004) encontraron que muchos árboles tenían una variedad de usos. Seis de las 20 especies registradas eran utilizadas como alimento, para artesanías manuales y/o remedios medicinales. Chazdon y Coe (1999) registraron 459 especies leñosas en comunidades de vegetación secundaria de las tierras bajas del Atlántico de Costa Rica. $70 \%$ de ellas tenía por lo menos un uso. La mayor riqueza de especies fue para las plantas medicinales (167 especies). En Venezuela, Aranguren (2005) encontró que de las 287 plantas útiles registradas (incluyendo herbáceas) la mayor cantidad corresponde a las medicinales, seguidas por las maderables, comestibles y de usos artesanales (de madera y cestería). Casas et al. (2001) en el Valle de Tehuacán, registraron 22 categorías de usos para 808 especies. Un total de 706 especies de plantas silvestres, arvenses y ruderales son forrajeadas tanto por humanos como por animales domésticos en las áreas donde se encuentran, aunque resalta que 59 de ellas son también manejadas in situ. Este manejo 
habla de un conocimiento y manipulación que va mucho más allá de la recolección. Entre las especies con más categorías de usos están Acacia farnesiana (11 usos), Guazuma ulmifolia (11 usos) y Pithecellobium dulce (10 usos).

Uno de los usos más comunes reportado en la literatura para algunas de las especies de selvas bajas y acahuales es la utilización del follaje como complemento de forraje o como abono verde. Las cualidades nutritivas de las hojas o frutos de algunos árboles son aprovechadas en la alimentación de los animales domésticos. En la zona no es frecuente este tipo de aprovechamiento, aunque en otras épocas la almendra y el follaje del ojite (Brosimum alicastrum) sirvió de forraje para el ganado y puercos. En Tehuacán, Camargo-Ricalde y Dhillion (2004), trabajando con el género Mimosa, mostraron que $45 \%$ de las especies se utilizó como forraje, 31\% como leña, $14 \%$ como cercas vivas, $7 \%$ para materiales de construcción y solamente una especie con fines medicinales. En otros países destaca el uso de plantas como forraje, tal es el caso de Leucaena leucocephala en Australia (Middleton et al., 1995), Gliricidia sepium en Asia sudoriental (Stewart, 1996), Sesbania grandiflora en Indonesia (Gutteridge, 1994), Gliricidia sepium, Brosimum alicastrum y Guazuma ulmifolia en Jamaica (Morrison et al., 1996) entre otros muchos trabajos. En regiones como Centroamérica hay experiencias importantes de este tipo, que no se llevan a cabo en la región de trabajo y que valdría la pena valorar e introducir.

En otras regiones hay experiencias interesantes sobre el uso de algunas de las especies enlistadas en este trabajo. Por ejemplo Gliricidia sepium se entremezcla con plantaciones de Terminalia amazonia para restaurar cobertura sin necesidad de fertilizar (Nichols et al.,
2001), o para buscar la complementariedad entre árboles (Gliricidia sepium entre otros) y cultivos para incrementar el rango de fenologías foliares y reducir la competencia por agua en prácticas agroforestales (Broadhead et al., 2003). También se ha buscado inducir el crecimiento de Enterolobium cyclocarpum y Cedrela odorata en potreros en los que se ha removido el ganado y añadido fertilizantes (Griscom et al., 2005). En México ya existen experiencias de reforestación de 15 años, particularmente en Nuevo León, con Enterolobium cyclocarpum y Gliricidia sepium (Foroughbakhch et al., 2006), entre otras. Estas experiencias deben aprovecharse para combinar las plantaciones forestales con la ganadería y fomentar el uso del follaje de plantas nutritivas para el alimento de animales, así como para diseñar sistemas de reforestación así como plantaciones más integrales, en los cuales el valor ambiental se mantenga.

No quisiéramos dejar de mencionar la concepción arraigada en la zona de que el fruto del guanacaste (Enterolobium cyclocarpum) ingerido por los animales les llega a producir la muerte. Esta información contrasta con la literatura donde hay abundantes referencias a la ingestión y dispersión de esta semilla por caballos y vacas y a su uso como alimento del ganado (Niembro, 1986; Ezenwa, 1998; Carranza-Montaño et al., 2003). Este es un aspecto que valdría la pena investigar con mayor detenimiento.

A pesar de que un porcentaje de los entrevistados ya casi no hace uso de las plantas, la mayoría sí lo hacen. La extracción sostenida e intensiva y concentrada en sólo algunas especies puede afectar de manera irreversible a los árboles, por ejemplo, con la extracción total de las raíces, el desprendimiento perimetral de las cortezas que impide la nutrición de las raíces y mata los árboles o, de plano su 
derribo total (Hersch-Martínez, 1997). Los pobladores perciben una disminución progresiva en la disponibilidad de diversas especies, particularmente las requeridas con una demanda creciente externa (cedro, caoba), pero también entre las que se usan localmente.

El mayor número de especies utilizadas para los usos más mencionados por los pobladores entrevistados, corresponde a los usos de leña, construcción, obtención de postes, plantas comestibles y medicinales, construcción de muebles y cercas vivas. Estos usos emplean y por lo tanto afectan al mayor número de especies, ya sea por tala completa o recolección de partes. Un programa de reforestación debería contemplar las especies más empleadas para los usos que constituyen aún parte importante de la vida de los pobladores.

La comercialización de las especie sútiles no es una práctica habitual. Muy pocas tienen valor comercial como el cedro, y por tanto ésta es la que más se comercializa, o bien cuando hay una necesidad grande de adquirir leña. En la literatura hay pocos registros de la comercialización de especies útiles por las comunidades locales. Un caso estudiado es el de Ceiba aesculifolia en el Valle de Tehuacán, para el cual se comercializan las semillas (Avendaño et al., 2006). Otro ejemplo se registró en la Reserva de la Biosfera Maya en Guatemala donde se identificaron más de 80 especies de árboles útiles y se consideró que $39 \%$ de estas especies útiles de árboles eran comerciables, en comparación con sólo $18 \%$ en la localidad de Uaxactún (Mutchnick y Mccarthy, 1997).

En la zona, las especies se reconocen fundamentalmente por su uso utilitario, pero también unas pocas por los servicios ambientales que prestan, destacando su uso como fijadoras de médanos o cortinas rompevientos y como alimento y refugio para flora y fauna. Este tipo de percepciones puede interpretarse como un incremento del componente ético en el ámbito del aprovechamiento de los recursos maderables. El Proyecto de Plan de Manejo La Mancha-El Llano (MorenoCasasola et al., 2006; Paradowska, 2006), desde hace una década presente en la zona, y sus acciones orientadas hacia la conservación, que incluyen la concienciación y educación ambiental, la protección comunitaria de especies en veda y la promoción de proyectos productivos sustentables, han logrado modificar actitudes en algunos sectores de la población.

En este trabajo sobre el uso de las especies arbóreas y palmas de las dunas costeras de Actopan, destaca el hecho de que los habitantes tienen una historia de poblamiento reciente que data de mediados del siglo pasado (Paradowska, 2006), y conocen y usan más de 55 especies de árboles (especies identificadas). Este número de especies representa $53 \%$ de las 103 especies arbóreas reportadas por Castillo-Campos y Travieso-Bello (2006) para las selvas bajas caducifolias de la zona, tanto sobre dunas como en los cerros. Es de notar que solamente 18 especies fueron mencionadas por más de cinco personas, lo cual muestra un reducido número de especies ampliamente reconocidas por su utilidad. Ello puede deberse a que muchos de los usos se vuelven cada vez más raros o han desaparecido de la práctica cotidiana, permaneciendo sólo en la memoria de los más viejos. En contraste, vale la pena resaltar la diferencia con respecto a los datos de Rico-Gray et al. (1991) tanto con respecto al alto número de especies que una comunidad maya utiliza y maneja como a los usos, siendo los más importantes las plantas medicinales, apícolas y alimenticias. Lo mismo puede decirse sobre el uso, manejo y domesticación de 
las especies del Valle de Tehuacán reportado en numerosos trabajos (Casas et al., 1999; 2001). Estos son usos que en las comunidades del municipio de Actopan son bajos y tienden a desaparecer (o apenas se empiezan a adquirir, como los apícolas).

Las restricciones impuestas por la ley al aprovechamiento de los recursos forestales fueron mencionadas con mayor frecuencia como una limitante para el uso de árboles de dunas. La prohibición de cortar árboles, independientemente de la tenencia del suelo en el que crece, es conocida comúnmente. Esta actitud contrasta con la del pasado, pero también significa que se deben dar pasos para incrementar la propagación, siembra y reforestación con las especies más usadas, y como segunda etapa iniciar las plantaciones forestales.

En síntesis, hay un conjunto de especies ampliamente mencionadas tanto por la diversidad de usos que tienen, porque son muy demandadas y/o porque se usan varias partes de la planta. A partir de este trabajo se considera que se debían usar para reforestar las dunas y enriquecer los acahuales que ya se están formando en los pastizales que cubren estos ecosistemas, así como impulsar y favorecer las cercas vivas con algunas de estas especies. Un siguiente paso será experimentar cuáles son las mejores condiciones para el establecimiento de dichas especies, sus requerimientos de agua y nutrimentos y su velocidad de crecimiento en estos ambientes. Las especies recomendadas son Diphysa robinioides, Gliricidia sepium, Cedrela odorata, Enterolobium cyclocarpum, Bursera simaruba, Tabebuia rosea, Guazuma ulmifolia, Brosimum alicastrum, Acacia cochliacantha, Maclura tinctoria, Spondias mombin, Karwinskia humboldtiana, Cordia dentata, Coccoloba barbadensis, Chrysobalanus icaco, Swietenia macrophylla y Acrocomia aculeata. Dos especies que no recibieron muchas menciones son Leucaena leucocephala y Sabal mexicana. La primera es usada en otras regiones como especie forrajera y la segunda cada vez sus hojas tienen una mayor demanda. Por tanto se considera que también se debían incluir entre el grupo de especies aptas para la reforestación de dunas. Las hojas de la palma mencionada ya se comercializan en la región, sobre todo para el techado de restoranes e infraestructura turística local y regional. Es una especie que puede proporcionar entradas económicas a los pobladores locales, por lo que también se considera apta para incluirse en un programa de reforestación.

La especie introducida, Casuarina equisetifolia, es apreciada localmente por los servicios ambientales que presta y como ornamental y su uso debe restringirse a formar cortinas rompevientos en ciertas situaciones. Otra especie útil localmente es Cocos nucifera por lo que también se sugiere su siembra, en zonas posteriores a la playa, más no en las dunas.

\section{CONCLUSIONES}

El presente trabajo muestra que el uso de los árboles sigue siendo vigente para necesidades básicas de la vida diaria, y que hay un conjunto de especies preferidas. Existe un pequeño grupo de especies arbóreas ampliamente utilizadas en la vida diaria. El uso de leña y de postes son dos usos vigentes, y en amplios sectores también la construcción, de tal modo que se requieren propuestas de reforestación que garanticen el abasto de madera. El uso de postes, impulsando las cercas vivas, puede traer asimismo beneficios ambientales. 
El mantenimiento del uso de los recursos naturales, en el rango racional y procurando equilibrar la demanda con prácticas de restauración, conservación y concienciación sobre la problemática ambiental, resulta muy importante porque fortalece el vínculo de las personas con los recursos que le ofrecen el sustento y ancla a los pobladores en su territorio, afianzando la identidad propia del grupo. Esta identidad fortalecida puede traducirse en actitudes positivas hacia lo que constituye su patrimonio cultural y natural. Finalmente, el conocimiento y los usos tradicionales de árboles de los médanos constituyen el patrimonio cultural del pueblo, vinculado profundamente al paisaje costero de Veracruz. Los cambios en los esquemas de uso de la tierra y los usos y costumbres ligados a ello, pueden significar la pérdida de especies y de la diversidad genética, especialmente de la biota tropical que fueron seleccionadas en función de los ambientes y microhábitats y que hoy perduran en ecosistemas estresantes como son las dunas costeras.

\section{RECONOCIMIENTOS}

A los habitantes de La Mancha, Palmas de Abajo y San Isidro por su hospitalidad y colaboración. Al proyecto CONAFORCONACYT núm. 14766 y el Instituto de Ecología A.C. (902-17). Agradecimientos especiales al Dr. Guillermo Ángeles y Graciela Sánchez-Ríos y un revisor anónimo, quienes enriquecieron este trabajo con sus valiosos comentarios y a don Enrique López Barradas, un inestimable interlocutor siempre.

\section{REFERENCIAS}

Acosta, P. R. 1986. La vegetación de la Sierra de Manuel Díaz, Ver., México. Tesis de Licenciatura. Facultad de Ciencias Biológicas, Universidad Veracruzana. Xalapa, Ver.
Aranguren, A. B. 2005. Plantas útiles empleadas por los campesinos de la región de Bailadores, Venezuela. Boletín Antropológico 23 (64): 139-165.

Avendaño S. y I. Acosta. 2000. Plantas utilizadas como cercas vivas en el estado de Veracruz. Madera y Bosques 6(1): 55-71.

Avendaño, A., A. Casas, P. Dávila y R. Lira. 2006. Use forms, management and commercialization of "pochote" Ceiba aesculifolia (H.B. \& K.) Britten \& Baker f. subsp. parvifolia (Rose) P. E. Gibbs \& Semir (Bombacaceae) in the Tehuacán Valley, Central Mexico. Journal of Arid Environments 67: 15-35

Brummitt, R. K. y C. E. Powell (eds). 1992. Authors of plant names. Royal Botanic Gardens, Kew. Londres. 731 p.

Bullock, H., A. Money y E. Medina. 1995. Seasonally dry tropical forest. Cambridge University Press. Cambridge, Gran Bretaña, 303 p.

Broadhead, J. S., C. K. Ong y C. R. Black. 2003. Tree phenology and water availability in semi-arid agroforestry systems. Forest Ecology and Management 180: 61-73.

Bye, R. A. 1993. The role of humans in the diversification of plants in Mexico. In: T. P. Ramamoorth, R. Bye, A. Lot y J. Fa. (eds.). Biological diversity in Mexico. Oxford University Press, Nueva York, pp. 707-731.

Caballero, J. y L. Cortés. 2001. Percepción, uso y manejo tradicional de los recursos vegetales en México. In: $\mathrm{B}$. Rendón Aguilar, S. Rebollar, J. Caballero y M. A. Martínez-Alfaro (eds.). Plantas, cultura y sociedad: estudio sobre la relación entre seres humanos y plantas en los albores del siglo XXI. 
Universidad Autónoma Metropolitana, Unidad Iztapalapa. SEMARNAT, México, D.F., pp. 79-100.

Camargo-Ricalde, S.L. y S.S. Dhillion. 2004. Use and management of Mimosa species in the TehuacánCuicatlán Valley, a tropical semi-arid region in Mexico (Fabaceae-Mimosoideae). Revista de Biología Tropical 52 (4): 845-851.

Carranza-Montaño, M., M. SánchezVelásquez, R. Pineda López y G. R. Cuevas. 2003. Forage quality and potential of species from the sierra de Manantlan (Mexico) tropical dry forest. Agrociencia 37 (2): 203-210.

Casas, A., J. L. Viveros y J. Caballero. 1994. Etnobotánica mixteca: sociedad, cultura y recursos naturales en la montaña de Guerrero. Consejo Nacional de la Cultura y las Artes e Instituto Nacional Indigenista, México, D.F., México, 366 p.

Casas, A., J. Caballero y A. ValienteBanuet. 1999. Use, management and domestication of columnar cacti in South-central Mexico: a historical perspective. Journal of Ethnobiology 19(1): 75-95.

Casas, A., A. Valiente-Banuet, J. L. Viveros, J. Caballero, L. Cortés, P. Dávila, R. Lira and I. Rodríguez. 2001. Plant resources of the Tehuacán-Cuicatlán Valley, Mexico. Economic Botany 55(1): 129-166.

Castillo-Campos, G. 2006. Las selvas. In: P. Moreno-Casasola (ed.). Entornos Veracruzanos: la costa de La Mancha. Instituto de Ecología A.C. ,Xalapa, pp. 221-230.

Castillo-Campos, G. y M. E. Medina. 2002. Árboles y arbustos de la Reserva Natural de La Mancha,
Veracruz. Instituto de Ecología A.C., Xalapa, 144 p.

Castillo-Campos, G. y A. C. TraviesoBello. 2006. La flora. In: P. MorenoCasasola (ed.). Entornos Veracruzanos: la costa de La Mancha. Instituto de Ecología, A.C., Xalapa, pp. 171-204.

Chazdon, R. L. y F. G. Coe. 1999. Ethnobotany of woody species in secondgrowth, old-growth, and selectively logged forests of Northeastern Costa Rica. Conservation Biology 13(6): 1312-1322.

Cronquist, A. 1988. The evolution and classification of flowering plants. New York Botanical Garden Press, Nueva York, 555 p.

Ezenwa, I. 1998. Preliminary evaluation of the suitability of Enterolobium cyclocarpum for use in intensive feed garden in southwestern Nigeria. Agroforestry Systems 44(1): 13-19

Foroughbakhch, R., M. A. AlvaradoVázquez, J. L. Hernández-Piñero, A. Rocha-Estrada, M. A. Guzmán-Lucio y E. J. Treviño-Garza. 2006. Establishment, growth and biomass production of 10 tree woody species introduced for reforestation and ecological restoration in northeastern Mexico. Forest Ecology and Management 235: 194-201.

García Gil, G. 2006. El ambiente geomorfológico. In: P. Moreno-Casasola (ed.). Entornos Veracruzanos: la costa de La Mancha. Instituto de Ecología A.C., Xalapa, pp. 1155-126.

Geissert, D. K. 2006. Procesos y cambios. In: P. Moreno-Casasola (ed.). Entornos Veracruzanos: la costa de La Mancha. Instituto de Ecología A.C., Xalapa, pp. 127-138. 
Griscom, H. P., M. S. Ashton y G. P. Berlyn. 2005. Seedling survival and growth of native tree species in pastures: Implications for dry tropical forest rehabilitation in central Panama. Forest Ecology and Management 218: 306-318.

Gustad, G., S. S. Dhillion y D. Sidibi. 2004. Local use and cultural and economic value of products from trees in the parklands of the municipality of Cinzana, Mali. Economic Botany 58(4): 578-587.

Gutteridge, R. C. 1994. The perennial Sesbania species. In: R. C. Gutteridge y $\mathrm{H}$. M. Shelton (eds.). Forage tree legumes in tropical agriculture. Wallingford, U.K., CAB International, pp. 49-64.

Hersch-Martínez, P. 1997. Medicinal plants and regional traders in Mexico: physiographic differences and conservational challenge. Economic Botany 51(2): 107-120.

Luoga, E. J., E. T. F. Witkowski y K. Balkwill. 2000. Differential utilization and ethnobotany of trees in Kitulanghalo Forest Reserve and surrounding communal lands, Eastern Tanzania. Economic Botany 54(3): 328-343.

Martínez, M. L., A. Intralawan, G. Vázquez, $\mathrm{O}$. Pérez-Maqueo, $\mathrm{P}$. Sutton, R. Landgrave. 2007. The coasts of our world: Ecological, economic and social importance. Ecological Economics 63: 254-272.

Masera, O. R., M. J. Ordóñez y R. Dirzo. 1997. Carbon emissions from Mexican forests: current situation and long-term scenarios. Climatic Change 35(3): 265-295

Mejía R. y S. A. Sandoval (coords.). 2003. Tras las vetas de la investigación cualitativa. Perspectivas y acercamiento desde la práctica. ITESO, México, D. F.

Middleton, C. H., R .J. Jones, H. M. Shelton, S. R. Petty y J. H. Wildin. 1995. Leucaena in northern Australia. In: H.M. Shelton, C. M. Piggin y J. L. Brewbaker (eds.). Leucaena -opportunities and limitations. ACIAR Proceedings núm. 57. Canberra, Australia, Australian Centre for International Agricultural Research. Bogor, Indonesia, pp. 214-221.

Moreno-Casasola, P. y A. C. TraviesoBello. 2006. Las playas y dunas. In: P. Moreno-Casasola (ed.). Entornos Veracruzanos: la costa de La Mancha. Instituto de Ecología A. C., Xalapa, pp. 205-220.

Moreno-Casasola, P. y G. Vázquez. 2006. Las comunidades de las dunas. In: P. Moreno-Casasola (ed.). Entornos Veracruzanos: la costa de La Mancha. Instituto de Ecología A. C., Xalapa, pp. 285-310.

Moreno-Casasola, P., G. Salinas, L. Amador, A. Juárez, H. H. Cruz, A. C. Travieso, L. Ruelas-Monjardín, R. Monroy, D. Infante, H. López, L. A. Peralta, K. Paradowska y A. Valencia. 2006. El proyecto comunitario de conservación y producción. In: P. Moreno-Casasola (ed.). Entornos Veracruzanos: la costa de La Mancha. Instituto de Ecología A. C., Xalapa, pp. 493-538.

Morrison, B. J., M. A. Gold y D. O. Lantagne. 1996. Incorporating indigenous knowledge of fodder trees into small-scale silvopastoral systems in Jamaica, Agroforestry Systems 34(1): 101-117. 
Mutchnick, P. A. y B. C. Mccarthy. 1997. An ethnobotanical analysis of the tree species common to the subtropical moist forests of the Peten, Guatemala. Economic Botany 51(2): 158-183.

Nichols, J. D., M. E. Rosemeyer, F. L. Carpenter y J. Kettler. 2001. Intercropping legume trees with native timber trees rapidly restores cover to eroded tropical pasture without fertilization. Forest Ecology and Management 152(1-3): 195-209.

Niembro, A. 1986. Árboles y arbustos útiles de México. Limusa, México, D. F. 206 p.

Paradowska, K. 2006. El poblamiento y el territorio. In: P. Moreno-Casasola (ed.). Entornos Veracruzanos: la costa de La Mancha. Instituto de Ecología, A.C., Xalapa, pp 35-64.

Pennington, T. D. y J. Sarukhán. 1998. Árboles tropicales de México. Manual para la identificación de las principales especies. UNAM-FCE. México, D.F., 521 p.

Pinedo-Vásquez, M., D. Zarin, P. Jipp y J. Chota-Inuma. 2005. Use-values of tree species in a Communal Forest Reserve in Northeast Peru. Conservation Biology 4(4): 405-416.

Rico-Gray, V., A. Chemás y S. Mandujano. 1991. Uses of tropical deciduous forest species by the Yuca- tecan Maya. Agroforestry Systems 14(2): 149-161

Ruelas-Monjardín, L. 2006. La situación socioeconómica. In: P. MorenoCasasola (ed.). Entornos Veracruzanos: la costa de La Mancha. Instituto de Ecología, A. C., Xalapa, pp. 65-90.

Rzedowski, J. 1978. La vegetación de México. Limusa S. A., México, D. F., $432 \mathrm{p}$.

Rincón, E., M. Álvarez, G. González, P. Huante y A. Hernández. 1999. Restauración de selvas bajas caducifolias en México. Gaceta Ecológica núm. 53, INE-SEMARNAP, pp. 62-71.

Stewart, J. L. 1996. Utilization. In: J. L. Stewart, G. E. Allison y A. J. Simons (eds.). Gliricidia sepium: genetic resources for farmers. Tropical Forestry Papers núm. 33, Oxford Forestry Institute. Oxford, pp. 33-48.

Travieso-Bello, A. C. y A. Campos. 2006. Los componentes del paisaje. In: $\mathrm{P}$. Moreno-Casasola (ed.). Entornos Veracruzanos: la costa de La Mancha. Instituto de Ecología, A.C., Xalapa, pp. 139-150.

Valles, M. S. 1997. Técnicas cualitativas de investigación social. Reflexión metodológica y práctica profesional. Editorial Síntesis, Madrid, España. $430 \mathrm{p}$. 
Anexo 1. Cuestionario utilizado para recabar la información sobre especies útiles de dunas costeras en las poblaciones de San Isidro y Colonia La Mancha en el municipio de Actopan

\section{Bloque 1. Datos del entrevistado}

1. Nombre del entrevistado

2. Localidad

3. Actividad productiva a que se dedica

\section{Bloque 2. Utilidad de médanos}

1. ¿Qué utilidad tienen los médanos para usted?

2. ¿Qué beneficio personal le brindan?

3. Dígame 15 plantas del médano que considere importantes:

\begin{tabular}{lccc}
\hline & \multicolumn{3}{c}{ Forma de vida } \\
\hline & hierba & arbusto & árbol \\
\hline & & & \\
1. & & & \\
\hline
\end{tabular}

2.

4. Dígame 10 árboles o palmas del médano que usted utilice.

\section{Bloque 3. Caracterización de las especies útiles (árboles y palmas)}

Respecto a cada especie mencionada, díganos:

1. ¿Con qué otro nombre la conoce?

2. ¿Para qué usa el árbol?

3. ¿Qué parte o partes usa?

4. ¿Por qué?

5. ¿Qué tan frecuente lo usa?

6. ¿Lo puede comprar?

7. ¿Cuánto vale? 


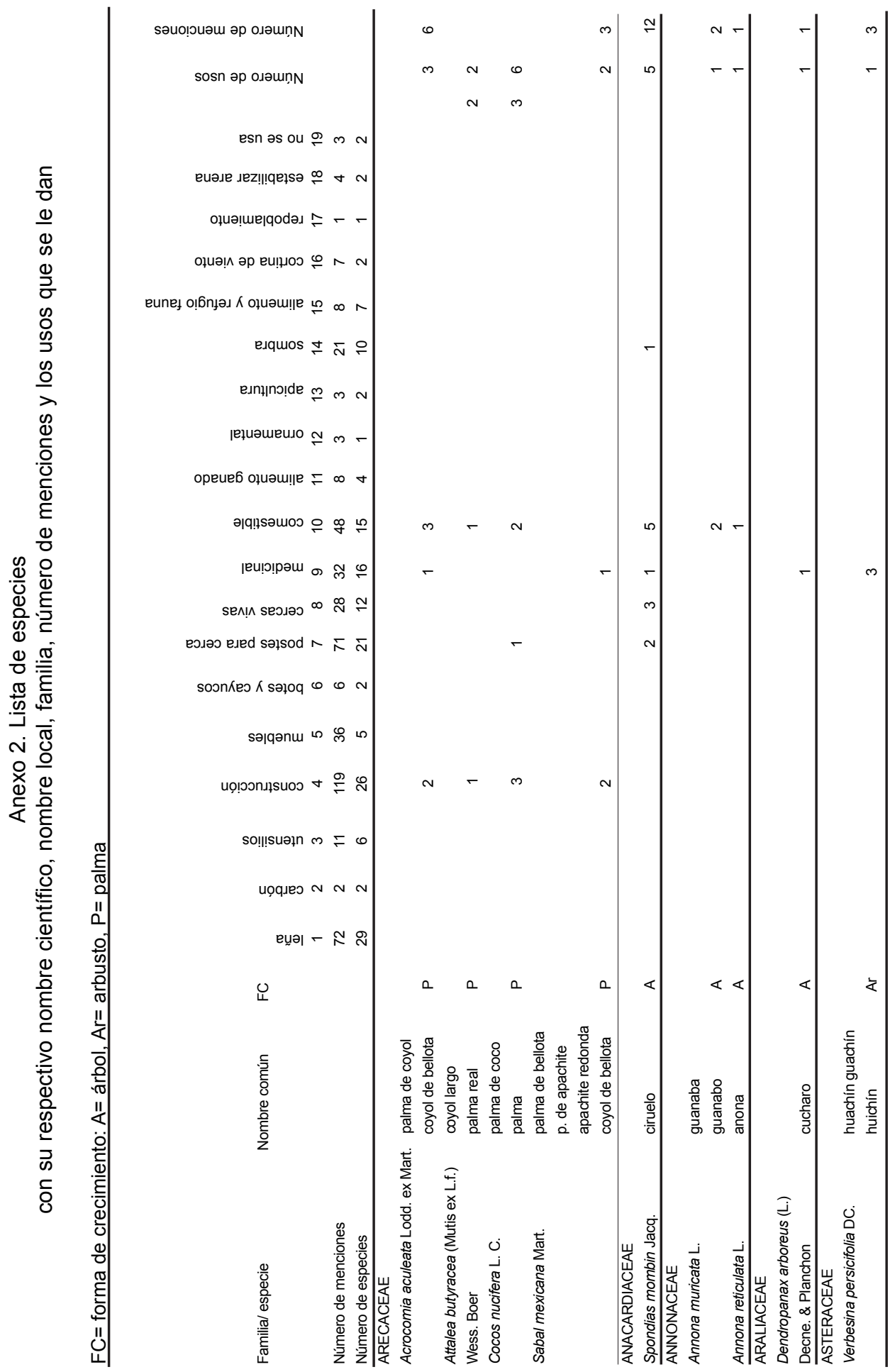




$$
\text { thi }
$$




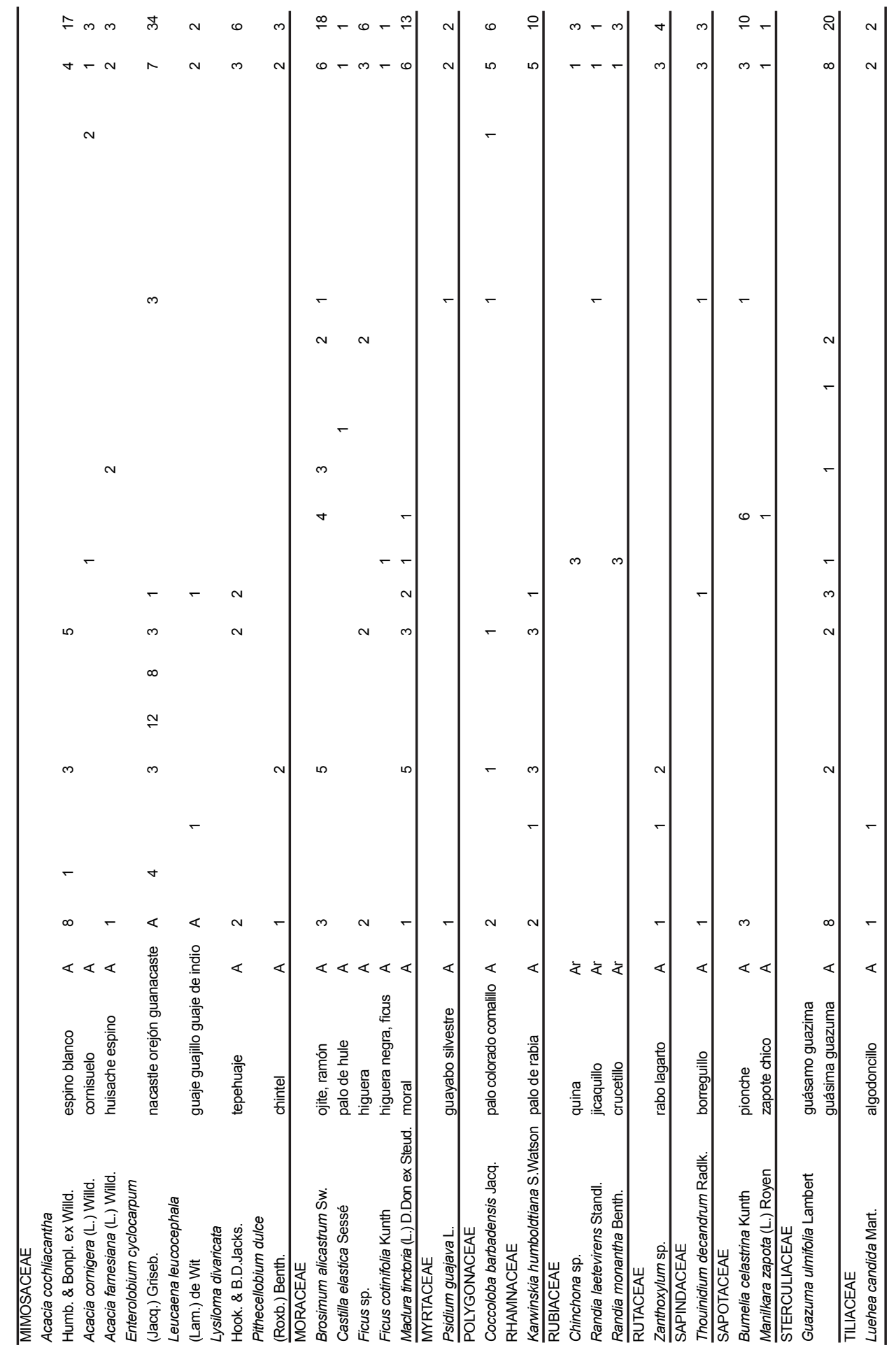

